\title{
Cross-species Discovery of Flubendazole against Melanoma Progression via MITF Downregulation and EMT Inhibition
}

\author{
Genta Kinoshita ${ }^{1}$, Eiji Yamada ${ }^{1,2}$, Hiroko Nakayama ${ }^{3}$, Richard M White ${ }^{4}$, Ewa B Snaar-Jagalska ${ }^{5}$, \\ Herman P Spaink ${ }^{5}$, Yasuhito Shimada ${ }^{1,2,6}$ \\ ${ }^{1}$ Integrative Pharmacology, Mie University Graduate School of Medicine, Japan, ${ }^{2}$ Mie University Zebrafish Anticancer \\ Drug Screening Center, Japan, ${ }^{3}$ Graduate School of Regional Innovation Studies, Mie University, Japan, ${ }^{4}$ Memorial \\ Sloan Kettering Cancer Center, USA, ${ }^{5}$ Department of Molecular Biology, Institute of Biology, Leiden University, the \\ Netherlands, ${ }^{6}$ Department of Bioinformatics, Mie University Advanced Science Research Promotion Center, Japan
}

Background: The incident rate of melanoma is increasing worldwide. New anti-melanoma medicines, such as BRAF inhibitor and anti-CTLA-4 antibody, improve 1-year survival rate to $60 \%$, while total survival duration is not prolonged. To discover new anti-melanoma drugs, we performed chemical screening using melanoma allograft zebrafish and discovered that flubendazole (FLU) reduced cancer burden.

Methods: We tested the anti-melanoma efficacy of FLU in vitro (culture cells) and in vivo. For in vitro, we performed cell proliferation assay with several kinds of melanoma cells (zebrafish ZMEL1, mouse B16F10 and human A375, COLO679, HMV-II and DEOC-1). For in vivo, we injected A375 cells into the circulation of zebrafish, and nude mice subcutaneously. To clarify the anti-melanoma mechanism of FLU, we performed the gene expression analysis for epithelial-mesenchymal transition (EMT) markers using qPCR. In addition, we conducted microphthalmia-associated transcription factor (MITF) knockdown in FLU-sensitive and resistant melanoma.

Results: FLU inhibited A375 cell proliferation in vitro $(\mathrm{IC} 50=125 \mathrm{nM})$ and in zebrafish $(\mathrm{IC} 50=358 \mathrm{nM})$. FLU $(25 \mathrm{mg} / \mathrm{kg}$ BW) also suppressed cancer proliferation in A375-mice. Interestingly, in zebrafish, FLU reduced cancer burden in caudal hematopoietic tissue, indicating that FLU could suppress extravasation. qPCR analyses revealed that FLU upregulated epithelial markers (CDH1 and ZEB2) except MITF, with downregulation of mesenchymal markers (VIM, ZEB1 and TWIST1) in zebrafish and mice. Contrary to other epithelial markers, MITF expression was suppressed by FLU. From in vitro study, we found some melanoma cell lines were resistant to FLU, and FLU could not downregulate MITF in these cells. In addition, MITF siRNA suppressed cell proliferation only in FLU-sensitive cells, not in FLU-resistant ones.

Conclusions: FLU suppressed melanoma progression via MITF and EMT inhibition. Integrated cross-species analysis using zebrafish, mice and human cells revealed that FLU should be one of strong candidates to inhibit tumor proliferation and metastasis. We further investigate the upstream mechanisms of FLU-induced MITF downregulation, which would be critical for FLU-sensitivity in individual melanomas. 